\title{
Unnötige Organisation oder sinnvolles Netzwerk einer neuen Generation von Hausärzten*?
}

Michael F. Bagattini

* Dieser Begriff (Hausärzte) gilt im gesamten Artikel immer für beide Geschlechter.

** Das «Vasco da Gama Movement» ist eine Europäische Jungärztebewegung, entstanden aus einer Arbeitsgruppe der Wonca Europe siehe auch Artikel von M. Reber Feissli und Reto J. D. Keller [1].

Korrespondenz:

med. pract. Michael F. Bagattini

Regensdorferstrasse 81

CH-8049 Zürich

Tel. 0439601094

michael.bagattini@gmx.ch

www.jhas.ch

\begin{abstract}
Ursprung
Anlässlich des SGAM-Kongresses von 2006 wurde im Jungärzteforum die Idee präsentiert, eine neue Hausärzteorganisation für junge und angehende Hausärzte, zu gründen. Zwei engagierte Hausärzte die ihren Schritt in die eigene Praxis erst in den letzten Jahren gewagt hatten, berichteten vom Vasco da Gama Movement** und ihren Plänen, in der Schweiz eine Jungärzteorganisation zu gründen. Auch ich stellte mir die Frage, weshalb in der Schweiz eine weitere Ärzteorganisation benötigt wird. Gibt es nicht bereits genügend Organisationen, in denen man sich einbringen kann und die unsere Anliegen abdecken? Schnell wurde jedoch klar, dass unter den verbliebenen ca. zehn angehenden oder jungen Hausärztinnen Einigkeit über die Notwendigkeit einer solchen Organisation herrschte: einer neuen Organisation, welche die Wünsche, Probleme und v.a. die Vernetzung der jungen und angehenden Hausärzte unterstützt.
\end{abstract}

\section{Ausgangslage und Probleme der jetzigen Weiterbildung}

Während die Weiterbildung der Assistenzärzte anderer Fachrichtungen an den stationären Einrichtungen meist mehr oder weniger gut strukturiert ist, sind die angehenden Allgemeinmediziner zumeist auf sich selbst angewiesen. Es werden Weiterbildungscurricula innerhalb einer Klinik durchlaufen, den geeigneten Kandidaten werden Anschlusslösungen an anderen Institutionen vermittelt und fachspezifische Weiterund Fortbildungskurse ermöglicht (häufig auch finanziell unterstützt), um das FMH-Weiterbildungscurriculum möglichst problemlos absolvieren zu können. Meistens ist der angehende Allgemeinmediziner der «Durchgangsassistent», der seine Jahre in verschiedenen Institutionen zusammensammelt und um dessen strukturierte Weiterbildung sich niemand kümmert. $\mathrm{Zu}$ viele Fragen sind hier offen, hinsichtlich der zu absolvierenden Jahre (seit 1. Juli 2006 zusätzlich neues FMH-Weiterbildungscurriculum) sowie der zu absolvierenden Kurse, und der Kontakt zu gleichgesinnten Fachassistenten ist schwierig. Aber auch nach abgeschlossener Weiterbildung zum Facharzt bestehen wenig strukturierte Angebote, die einem den Schritt in die eigene Praxis erleichtern.

\section{Société suisse des jeunes médecins} de premier recours: organisation inutile ou réseau judicieux d'une nouvelle génération de généralistes?

Lors du congrès de la Société suisse de médecine générale (SSMG) en 2006 à Bâle, un forum de jeunes médecins a émis des plans concrets de fondation d'une organisation de jeunes généralistes suisses. Pourquoi a-t-on besoin en Suisse, hormis toutes les sociétés médicales existantes, d'une nouvelle organisation? La formation postgraduée des médecins de premier recours en devenir est peu structurée et manque de réseaux. Les contacts et les échanges entre jeunes médecins sont lacunaires. Une organisation de jeunes médecins de famille a donc été créée afin de soutenir l'avenir de la relève des généralistes et d'établir un forum d'échange d'informations. Une première étape sera d'améliorer les liens et l'information entre les jeunes médecins de famille. Nous espérons ainsi augmenter l'attrait de la profession et en assurer I'identité. II va de soi que nous voulons et devons aussi faire en sorte que la nouvelle organisation s'implique et soit reconnue dans les processus politiques, afin de contribuer à forger à long terme l'image professionnelle du médecin de premier recours. II s'agit en effet de notre avenir et nous voulons prendre part à sa réalisation. 


\section{Engagement und Vernetzung}

Nach intensiven Vorbereitungen wurde die neue Organisation am 11. November 2006 ins Leben gerufen. Was möchte nun diese neue Organisation? Die jungen Hausärztinnen und -ärzte Schweiz - JHaS [2] - setzen sich für die jungen und zukünftigen Hausärzte ein. Primäres Ziel ist die Vernetzung und der Informationsaustausch unter den jungen Kolleginnen und Kollegen, um von den Erfahrungen jedes einzelnen zu profitieren und zu lernen. Die JHaS unterstützen sie in ihren Anliegen und Problemen und möchten sie in der beruflichen Identität fördern. Sie bieten ein Kommunikationsnetzwerk und eine Informationsplattform, setzen sich für die Teilnahme an nationalen und internationalen Hausarztkongressen und -weiterbildungen ein. Gemeinsam sollen Zukunftsvisionen und die Anliegen der zukünftigen Hausärztegeneration in den politischen Prozess eingebracht werden. Insbesondere soll die Hausarztmedizin bereits während des Studiums, vor allem aber auch während der Assistenzzeit gefördert werden. Die Vernetzung des Nachwuchses sowie seine Unterstützung und somit auch Förderung gehören also zu den Aufgaben, und wer könnte dies besser als junge Ärzte, die ihre Weiterbildungszeit eben erst beendet haben oder noch darin stekken? Der Schritt in die eigene Praxis wirft immer wieder die gleichen Fragen auf. Auch hier wollen die JHaS eine Vernetzung schaffen, damit nicht jedesmal das «Rad neu erfunden werden muss». Die Vernetzung ist überhaupt das wichtigste Instrument. Die Möglichkeit, neue Kontakte zu knüpfen und Informationen auf breiter Basis auszutauschen, spart Energien, fördert den Austausch und steigert die Qualität der Arbeit. Dabei wird auch über die Grenzen hinausgeschaut und durch die Zusammenarbeit mit dem Vasco da Gama Movement der Austausch auf internationaler Ebene gefördert. In Zeiten des Umbruchs müssen verschiedene Projekte, Modelle in den Entwicklungsprozess miteinbezogen werden, und da kann ein Blick in die Nachbarländer sicher nicht schaden.

\section{Zukunftsperspektive}

Zwar sind erfreulicherweise bereits viele Bestrebungen im Gange, die zukünftigen Grundversorger zu unterstützen. So gibt es das KHM-Projekt für Praxisassistenzen, es werden Praxiseröffnungskurse angeboten, Arbeitsabläufe angepasst wie beispielsweise die Umstrukturierung des Notfalldienstes usw., doch besteht weiterhin Handlungsbedarf auf breiter Ebene. Die JHaS möchten bestehende Defizite aufheben, indem sie eine Anlaufstelle für die jungen Ärzte bilden, die sich in den Gegebenheiten der verschiedenen Organisationen und Institutionen noch auf unsicherem Parkett bewegen, sie an die bestehenden Angebote heranführen sowie die bereits laufenden Bestrebungen unterstützen. Ebenso möchten die JHaS den Hausärztenachwuchs für das standespolitische Engagement sensibilisieren sowie die bestehenden Organisationen und Institutionen auf die Probleme der angehenden Grundversorger hinweisen. Sicherlich wird sich die neue Organisation auch auf politischer Ebene einbringen müssen, die Förderung der Hausarztmedizin bereits während des Studiums unterstützen und nicht zuletzt auch das Image der Hausärzte verbessern wollen, doch bis dahin ist noch ein weiter Weg zu beschreiten. Die ersten kleinen Schritte werden nun gemacht, denn nur so bewegt man sich. So gesehen ist die Gründung der JHaS sicher nicht einfach das Erstellen einer weiteren überflüssigen Organisation, sondern ein sinnvoller Beitrag zur Stärkung und Sicherung der Grundversorgung der schweizerischen Bevölkerung.

\section{Literatur}

1 Keller RJD, Reber Feissli M. Vom Vasco da Gama Movement zum JungärztInnenforum. PrimaryCare. 2006;6(45):832-4. Version française: Keller RJD, Reber Feissli M. Du Vasco da Gama Movement au forum pour jeunes médecins. PrimaryCare. 2006;6(46):857-9.

2 Reber Feissli M. Junge Hausärztinnen und -ärzte Schweiz - JHaS. PrimaryCare. 2007;7(1-2):26-7. 